\title{
Robust immune responses elicited by a fully synthetic three-component vaccine
}

\author{
Sampat Ingale, Margreet A Wolfert, Jidnyasa Gaekwad, Therese Buskas \& Geert-Jan Boons
}

\begin{abstract}
The overexpression of saccharides such as Globo-H, Lewis ${ }^{Y}$ and $\mathrm{Tn}$ antigen is a common feature of oncogenic transformed cells. Endeavors to exploit this aberrant glycosylation for cancer vaccine development have been complicated by difficulties in eliciting high titers of $\lg G$ antibodies against classical conjugates of tumor-associated carbohydrates to carrier proteins. We have designed, chemically synthesized and immunologically evaluated a number of fully synthetic vaccine candidates to establish strategies to overcome the poor immunogenicity of tumor-associated carbohydrates and glycopeptides. We have found that a three-component vaccine composed of a TLR2 agonist, a promiscuous peptide T-helper epitope and a tumor-associated glycopeptide can elicit in mice exceptionally high titers of $\operatorname{lgG}$ antibodies that can recognize cancer cells expressing the tumor-associated carbohydrate. The superior properties of the vaccine candidate are attributed to the local production of cytokines, upregulation of costimulatory proteins, enhanced uptake by macrophages and dendritic cells and avoidance of epitope suppression.
\end{abstract}

A broad and expanding body of preclinical and clinical studies ${ }^{1-4}$ demonstrates that naturally acquired, passively administered or actively induced antibodies against carbohydrate-associated tumor antigens are able to eliminate circulating tumor cells and micrometastases in individuals with cancer. Tumor-associated saccharides are, however, of low antigenicity, because they are self-antigens and consequently are tolerated by the immune system. In addition, foreign carrier proteins such as keyhole limpet hemocyanin (KLH) and bovine serum albumin (BSA) and the linker that attaches the saccharides to the carrier protein can elicit strong B-cell responses, which may lead to the suppression of antibody responses against the carbohydrate epitope ${ }^{5,6}$. It is clear that the successful development of carbohydratebased cancer vaccines requires new strategies for the more efficient presentation of tumor-associated carbohydrate epitopes to the immune system, which would result in a more efficient class switch to IgG antibodies ${ }^{7-17}$.

We reasoned that a three-component vaccine composed of a tumorassociated carbohydrate B epitope, a promiscuous peptide T-helper (Th) epitope and a Toll-like receptor (TLR) ligand would circumvent immune suppression caused by a carrier protein or the linker region of a classical conjugate vaccine. Such a vaccine candidate would contain, however, all mediators required for eliciting a strong IgG immune response. First, we designed vaccine candidates $\mathbf{1}$ and $\mathbf{2}$, which contain as a B epitope a tumor-associated glycopeptide derived from MUC1 (refs. 1,18) and the well-documented mouse major histocompatibility complex (MHC) class II restricted Th epitope 103-KLFAVWKITYKDT-115 derived from the polio virus ${ }^{19}$ (Fig. 1). Furthermore, compound 1 contains as a built-in adjuvant the lipopeptide $\mathrm{Pam}_{2}$ CysSK 4 , which is a potent activator of TLR2 and TLR6, whereas compound 2 contains $\mathrm{Pam}_{3} \mathrm{CysSK}_{4}$, which induces cellular activation through TLR1 and TLR2 (ref. 20).

Compound $\mathbf{1}$ was prepared by a solid-phase peptide synthesis protocol using a Rink amide AM resin (Novabiochem, EMD Biosciences), $N$-fluorenylmethoxycarbonyl ( $N^{\alpha}$-Fmoc)-protected amino acids and $N^{\alpha}$-Fmoc-Thr-( $\mathrm{AcO}_{3}-\alpha$-D-GalNAc) (3) ${ }^{21}$. After assembly of the glycopeptide, the acetyl esters of the saccharide moiety were cleaved by treatment with $80 \%$ hydrazine in methanol. Next, the resulting product was coupled manually with $N^{\alpha}$-Fmoc- $(S)$-(2,3-bis(palmitoyloxy)-(2R)-propyl)-( $R$ )-cysteine ( $N^{\alpha}$-Fmoc-Pam ${ }_{2}$ Cys-OH $) \quad(4)^{22}$, followed by removal of the $N^{\alpha}$-Fmoc group using piperidine $(20 \%)$ in $\mathrm{N}, \mathrm{N}$-dimethylformamide (DMF) and cleavage from resin using reagent B (trifluoroacetic acid, 88\%; phenol, 5\%; water, 5\%; triisopropylsilane, $2 \%)$ to give compound $\mathbf{1}$. Unfortunately, a similar linear synthesis of compound 2 gave a product that was difficult to purify to homogeneity. Therefore, 2 was prepared by liposome-mediated native chemical ligation (NCL) of building blocks $\mathbf{5 a}, \mathbf{6}$ and 7 (ref. 23). Thus, a film of dodecylphosphocholine, thiol $\mathbf{5 a}$ and thioester $\mathbf{6}$ was hydrated in a phosphate buffer ( $\mathrm{pH}$ 7.5) in the presence of carboxyethyl phosphine and EDTA and then ultrasonicated. The resulting vesicles were sized to $1 \mu \mathrm{m}$ by passing through a polycarbonate membrane filter. The ligation was initiated by the addition of sodium 2-mercaptoethane sulfonate, and LC-MS showed completion of the reaction after $2 \mathrm{~h}$. Homogenous glycopeptide 8a was obtained after removal of the acetamidomethyl protecting group of the ligation product using $\mathrm{Hg}$ (II) acetate and purification by RP-HPLC (Agilent Technologies) over a C4 column (Phenomenex). A second liposome-mediated NCL of the free sulfhydryl moiety of $\mathbf{8 a}$ with thioester $\mathbf{7}$ gave glycolipopeptide $\mathbf{2}$ in an excellent overall yield. For synthetic details see Supplementary Methods online.

Next, 1 and 2 were incorporated into phospholipid-based small unilamellar vesicles (SUVs) by hydration of a thin film of the 


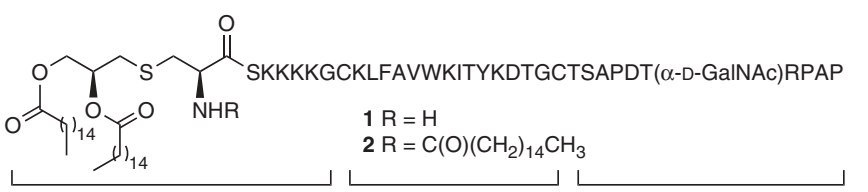

TLR ligand

Th epitope

B epitope

XTSAPDT $(\alpha-D$-GalNAc)RPAP $\quad 5 a \mathrm{X}=$ Cys

(B epitope) $\quad 5 \mathbf{b} X=\mathrm{H}$

$\mathrm{H}_{\text {SAcm }}^{\mathrm{H}_{2} \mathrm{~N}-\mathrm{CKLFAVWKITYKDTG} \prod_{\mathrm{O}}^{\mathrm{O}}} \underset{\text { (Th epitope building block) }}{\mathrm{SBn}}$

(TLR ligand building block)

XKLFAVWKITYKDTGXTSAPDT( $\alpha$-D-GaINAC)RPAP $\quad$ 8a $X=$ Cys

(Th/B epitope)

$8 \mathbf{b} X=\mathrm{H}$

SKKKK 9 (TLR ligand)

KLFAVWKITYKDT 10 (Th epitope)

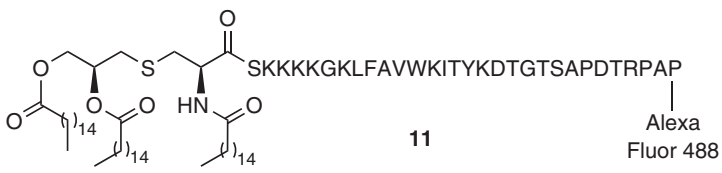

Figure 1 Structures of synthetic compounds. Acm, acetamidomethyl; Bn, benzyl.

synthetic compounds, egg phosphatidylcholine, egg phosphatidylglycerol and cholesterol in a HEPES buffer followed by extrusion through a $100 \mathrm{~nm}$ Nuclepore polycarbonate membrane (Millipore). Groups of five female BALB/c mice (Jackson Laboratories) were immunized intraperitoneally four times at weekly intervals with liposomes containing $3 \mu \mathrm{g}$ of saccharide. To explore the adjuvant properties of the vaccine candidates, liposomes were administered with or without the potent saponin immunoadjuvant QS-21 (ref. 24).

Anti-MUC1 antibody titers were determined by coating microtiter plates with CTSAPDT $(\alpha-D-G a l N A c) R P A P$ conjugated to bromoacetyl-modified BSA, and detection was accomplished with antimouse IgM and IgG antibodies labeled with alkaline phosphatase. Mice immunized with 2 elicited exceptionally high titers of antiMUC1 IgG antibodies (Table 1). Subtyping of the IgG antibodies indicated a bias toward a Th2 response ${ }^{25}$. Furthermore, the observed high IgG3 titer is typical of an anticarbohydrate response. Coadministration of adjuvant QS-21 did not lead to a significant increase of IgG antibodies; however, in this case a mixed Th1/Th2 response was observed. Surprisingly, the use of glycolipopeptide 1, which contains $\mathrm{Pam}_{2} \mathrm{CysSK}_{4}$ instead of $\mathrm{Pam}_{3} \mathrm{CysSK}_{4}$, gave lower titers of IgG antibodies. Compounds 1 and 2 elicited low titers of antibodies against the Th epitope, which indicates that the vaccine does not suffer from immune suppression.

To ensure that the mouse sera were able to recognize the native MUC1 antigen present on cancer cells, binding of the sera to the
MUC1-expressing MCF7 human breast cancer cell line was examined by flow cytometry. The antisera elicited against $\mathbf{1}$ and $\mathbf{2}$ reacted strongly with the MUC1-positive tumor cells, whereas no binding was observed when SK-MEL-28 cells, which do not express the MUC1 antigen, were used (Fig. 2). Further studies showed that both compounds induce the secretion of cytokines such as tumor necrosis factor- $\alpha$ (TNF- $\alpha)$ in a TLR2-dependent manner, thereby leading to the upregulation of costimulatory proteins such as CD80, CD83 and CD86 (Supplementary Figs. 1-3 online).

Uptake and proteolytic processing of antigen and subsequent presentation of the peptide Th epitope as a complex with MHC class II on the cell surface of antigen-presenting cells (APCs) is critical for eliciting IgG antibodies. Thus, immunizations with a liposomal preparation of the individual components of $\mathbf{2}$ does not require such processing and hence may lead to even more robust antigenic responses. The influence of covalent attachment of the various components of the vaccine candidate on antigenic responses was investigated by immunizing mice with a liposomal preparation of compound $\mathbf{8 b}$, which is composed of the Th epitope linked to the B epitope, and the adjuvant $\mathrm{Pam}_{3} \mathrm{CysSK}_{4}(9)$. Significantly lower $(P<$ $0.05)$ titers of IgG antibodies were found relative to those found when 2 was used (Table 1). Zero or very low IgG antibody responses were observed when the two compounds were administered as a saline solution, which indicates that a liposomal preparation contributes to antigenicity. The importance of covalent attachment was further highlighted in an experiment in which the B epitope (5b), Th epitope (10) and adjuvant 9 were administered as a liposomal preparation, which resulted in low titers of IgG antibodies.

It was anticipated that in addition to initiating the production of cytokines and stimulating the upregulation of costimulatory proteins, the lipopeptide $\mathrm{Pam}_{3} \mathrm{CysSK}_{4}$ may facilitate selective uptake by APCs in a TLR2-dependent manner. To test this hypothesis, compound 11, which contains an Alexa Fluor 488 fluorescence label, was prepared (Supplementary Methods) and administered to mouse macrophages. After $30 \mathrm{~min}$, the cells were harvested and lysed and the fluorescence was measured. To account for possible cell surface binding without internalization, the cells were also treated with trypsin before lyses and then examined for fluorescence. A substantial quantity of 11 was internalized, whereas a small amount was attached to the cell surface. To determine whether the uptake was mediated

Table 1 ELISA anti-MUC1 antibody titers after four immunizations with various preparations

\begin{tabular}{lrrrrrr}
\hline Immunization $^{\text {a }}$ & IgG total $^{\text {b }}$ & IgG1 & IgG2a & IgG2b & IgG3 & IgM \\
\hline $\mathbf{1}$ & 20,900 & 66,900 & 700 & 900 & 7,300 & 1,400 \\
$\mathbf{1}$ and QS-21 & 30,200 & 113,100 & 23,000 & 6,600 & 17,800 & 1,100 \\
$\mathbf{2}$ & 169,600 & 389,300 & 56,500 & 42,700 & 116,800 & 7,200 \\
2 and QS-21 & 322,800 & 371,300 & 378,900 & 56,800 & 263,500 & 5,000 \\
8b and $\mathbf{9}$ & 16,600 & 26,800 & 3,300 & 3,100 & 7,800 & 1,000 \\
8b and $\mathbf{9}$ (saline) & 600 & 400 & 400 & 800 & 400 & 400 \\
5b, 9 and $\mathbf{1 0}$ & 2,300 & 8,400 & 0 & 0 & 0 & 300 \\
\hline
\end{tabular}

Anti-MUC1 antibody titers are presented as the median for groups of five mice. ELISA plates were coated with BSA-BrAc-MUC1 conjugate and titers were determined by linear regression analysis, plotting dilution versus absorbance. Titers are defined as the highest dilution yielding an optical density of 0.1 or greater over that of normal control mouse sera.

aLiposomal preparations were used, except for $\mathbf{8 b}$ and $\mathbf{9}$ (saline). ${ }^{\mathrm{b}} \mathrm{A}$ statistically significant difference $(P<0.05)$ was observed between $\mathbf{1}$ versus $2, \mathbf{2}$ versus $8 \mathrm{~b}$ and $\mathbf{9}, \mathbf{2}$ versus $8 \mathrm{~b}$ and $\mathbf{9}$ (saline), $\mathbf{2}$ versus $5 \mathrm{~b}, \mathbf{9}$ and $\mathbf{1 0}$, and $\mathbf{8 b}$ and $\mathbf{9}$ versus $\mathbf{8 b}$ and $\mathbf{9}$ (saline). Individual titers for $\operatorname{lgG}$ total, IgG1, IgG2a, IgG2b, IgG3 and IgM are reported in Supplementary Figures $\mathbf{4}$ and $\mathbf{5}$ online. Titers after three and five immunizations for 2 and for $\mathbf{2}$ plus QS-21 are listed in Supplementary Table 1 online. 
LETTERS

Figure 2 Flow cytometry analysis for specific anti-MUC1 antibodies. (a,b) Reactivity was tested on MCF7 (a) and SK-MEL-28 (b) cells. Fluorescence intensity of serum (1:50 diluted) was assessed before (serum control; pink) and after four immunizations with 2 (orange). Also shown are medium (red) and conjugate (blue) controls. This is a representative example of the group of mice immunized with $2(n=5)$. Similar results were obtained for mice immunized with $\mathbf{2}$ in the presence of QS-21 and with 1 in the absence and presence of QS-21.
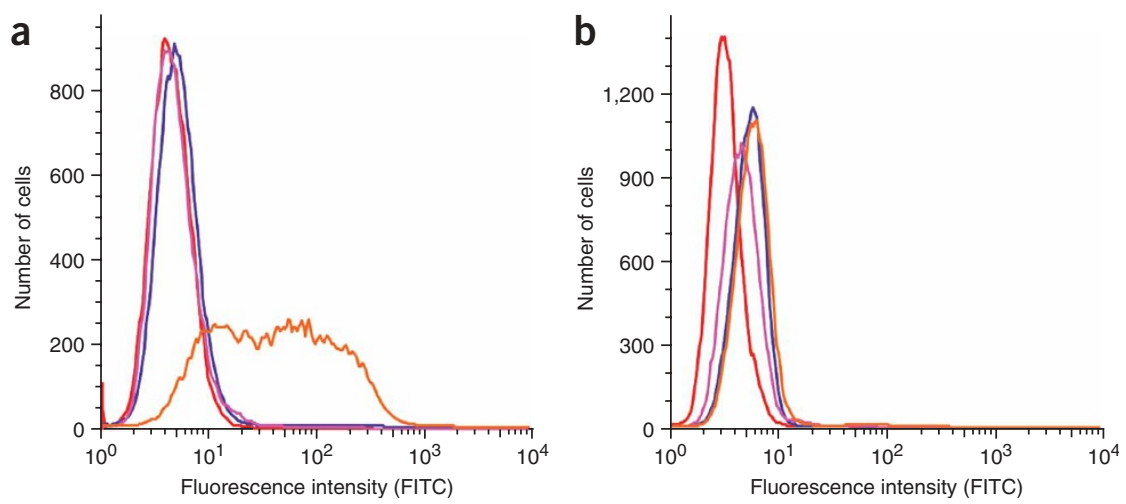

by TLR2, uptake studies were also performed using wild-type human embryonic kidney (HEK) 293T cells and HEK 293T cells stably transfected with mouse TLR2, TLR2 plus TLR6, or TLR4 plus CD14 and MD2 (Fig. 3). Importantly, substantial uptake was only observed when the cells were transfected with TLR2, which indicates that uptake is mediated by this receptor. It is important to note that macrophage galactose type C-lectin may also facilitate uptake of the vaccine candidates by binding to the Tn antigen, which is not probed by compound $11^{26}$.

Most efforts aimed at developing carbohydrate-based cancer vaccines have focused on the use of chemically synthesized tumorassociated carbohydrates linked through an artificial linker to a carrier protein $^{1-4}$. The use of KLH as a carrier protein in combination with the powerful adjuvant QS-21 gives the best results. However, a drawback of this approach is that KLH is a very large and cumbersome protein that can elicit high titers of anti-KLH antibodies, which leads to immune suppression of the tumor-associated carbohydrate epitope ${ }^{27}$. Furthermore, the conjugation chemistry is often difficult to control, which results in conjugates with ambiguities in composition. Also, the linker moiety can elicit strong B-cell responses ${ }^{5,6}$. Not surprisingly, preclinical and clinical studies with carbohydrate-protein conjugates have led to results of mixed merit. For example, mice immunizations with a trimeric cluster of Tn antigens conjugated to

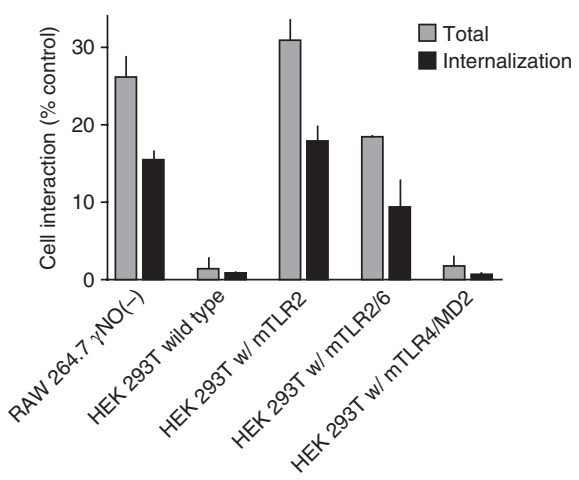

Figure 3 Cellular uptake of compound 11. Cells (RAW $264.7 \gamma$ NO(-), HEK 293T wild type and HEK 293T stably transfected with mouse TLR2, TLR2 plus TLR6 or TLR4 plus CD14 and MD2) were exposed to Alexa fluor 488-labeled compound $11\left(1 \mu \mathrm{g} \mathrm{ml}^{-1}\right)$ for $30 \mathrm{~min}$. After cells were washed and lysed (total cell interaction; gray) or washed, treated with trypsin and then lysed (internalization only; black), fluorescence (absorbance $485 \mathrm{~nm}$, emission $538 \mathrm{~nm}$ ) was measured. Fluorescence values were normalized for maximum possible fluorescence (100\%). Data represent mean values \pm s.d. $(n=3)$.
$\mathrm{KLH}$ in the presence of the adjuvant QS-21 elicits modest titers of IgG antibodies $^{28}$. In a clinical trial of individuals with relapsed prostate cancer, the vaccine gave low median IgG and IgM antibody titers ${ }^{29}$. In another study, only KLH conjugates of glycopeptides composed of multiple repeat units that are highly glycosylated with $\mathrm{Tn}$ antigens elicited IgG antibodies ${ }^{30}$.

The excellent antigenicity of the here-reported three-component vaccine is attributed to several features. First, it does not have any unnecessary components that are antigenic and may induce immune suppression. The chemical attachment of the TLR2 agonist Pam 3 CysSK $_{4}$ to the B and Th epitopes ensures that cytokines are produced at the site at which the vaccine interacts with immune cells, which leads to a high local concentration of cytokines, thereby facilitating maturation of relevant immune cells. It also facilitates uptake by TLR2-expressing cells such as APCs, which assist antigen processing and full activation. Finally, a fully synthetic approach makes it possible to optimize the various components of the candidate vaccine by structure-activity relationship studies. In this respect, proper design of the three-component vaccine is essential, because previously we showed that a compound composed of the inferior adjuvant $\mathrm{Pam}_{3} \mathrm{Cys}$ (which lacks the important tetralysine moiety) and a human Th epitope elicits low titers of $\operatorname{IgG}$ antibodies ${ }^{7,14}$. Furthermore, it has been found that covalent attachment of the three components is important for optimal antigenic responses. Probably, the lipid moiety of the vaccine candidate facilitates proper presentation and retention into liposomes. In this respect, liposomes can present the B epitopes multivalently, which facilitates B-cell receptor clustering and thus results in B-cell activation.

\section{METHODS}

General methods for solid-phase peptide synthesis. Peptides were synthesized by established protocols on an ABI 433A peptide synthesizer (Applied Biosystems) equipped with UV detector using $N^{\alpha}$-Fmoc-protected amino acids and 2-(1H-benzotriazole-1-yl)-1,1,3,3-tetramethylhexafluorophosphate (HBTU) plus $N$-hydroxybenzotriazole (HOBt) as the activating reagents. Single coupling steps were performed with conditional capping. The following protected amino acids were used: $N^{\alpha}$-Fmoc- $\mathrm{Arg}(\mathrm{Pbf})-\mathrm{OH}, N^{\alpha}$-Fmoc-Asp $(\mathrm{O}-t-\mathrm{Bu})-\mathrm{OH}$, $N^{\alpha}$-Fmoc-Cys(acetamidomethyl)-OH, $N^{\alpha}$-Fmoc-Cys(Trt)-OH, $N^{\alpha}$-Fmoc-Lys (Boc)-OH, $N^{\alpha}$-Fmoc-Ser $(t-\mathrm{Bu})-\mathrm{OH}, N^{\alpha}$-Fmoc-Thr $(t-\mathrm{Bu})-\mathrm{OH}$ and $N^{\alpha}$-Fmoc$\operatorname{Tyr}(t-\mathrm{Bu})-\mathrm{OH}$ (Pbf, $N^{\mathrm{G}}-(2,2,4,6,7-$ pentamethyldihydrobenzofuran-5-sulfonyl); Trt, trityl; Boc, $N$-t-butoxycarbonyl). $N^{\alpha}$-Fmoc groups were cleaved by piperidine (20\%) in DMF for $1 \mathrm{~h}$. The couplings of glycosylated amino acid $N^{\alpha}$-Fmoc-Thr- $\left(\mathrm{AcO}_{3}-\alpha\right.$-D-GalNAc) $(3)^{21}$ and $N^{\alpha}$-Fmoc-( $\left.S\right)$-(2,3-bis(palmitoyloxy)-(2R)-propyl)-( $R$ )-cysteine (4), which was prepared from $(R)$-glycidol ${ }^{22}$, were carried out manually using $O$-(7-azabenzotriazol-1-yl)- $N, N, N^{\prime}, N^{\prime}$-tetramethyluronium hexafluorophosphate (HATU) plus 1-hydroxy-7-azabenzotriazole (HOAt), and benzotriazole-1-yl-oxy-tris-pyrrolidinophosphonium (PyBOP) plus $\mathrm{HOBt}$, as coupling reagents, respectively. Progress of the manual 
couplings was monitored by standard Kaiser test. Compounds $\mathbf{1}, \mathbf{5 a}, \mathbf{5 b}, \mathbf{8 b}, \mathbf{9}$ and 10 were prepared on Rink amide AM resin, and thioesters 6 and 7 were synthesized on sulfamylbutyryl Novasyn TG resin (Novabiochem, EMD Biosciences). The preparation of 11 was carried out on universal NovaTag resin (Novabiochem, EMD Biosciences). Liposome-mediated NCL of $\mathbf{7}$ with $\mathbf{8 a}$ to give 2 was performed as described previously ${ }^{23}$. A similar procedure was used for the synthesis of compound $\mathbf{8 b}$ using $\mathbf{5 a}$ and $\mathbf{6}$. Peptides, glycopeptides, lipopeptides and glycolipopeptides were purified by HPLC using semipreparative C4 and C8 columns and linear gradients of 0 to $95 \%$ solvent B $(0.1 \%$ trifluoroacetic acid (TFA) in acetonitrile) in solvent A (0.1\% TFA in water). Details of the chemical synthesis and analytical data of the compounds are provided in the Supplementary Methods.

General procedure for the preparation of liposomes. Egg phosphatidylcholine, phosphatidylglycerol, cholesterol and synthetic compound (15 $\mu \mathrm{mol}$, molar ratios, 65:25:50:10) were dissolved in a mixture of trifluoroethanol and $\mathrm{MeOH}(1: 1, \mathrm{v} / \mathrm{v}, 5 \mathrm{ml})$. The solvents were removed in vacuo to give a thin lipid film, which was hydrated by shaking in HEPES buffer ( $10 \mathrm{mM}, \mathrm{pH} 6.5)$ containing $\mathrm{NaCl}(145 \mathrm{mM})(1 \mathrm{ml})$ under argon atmosphere at $41^{\circ} \mathrm{C}$ for $3 \mathrm{~h}$. The vesicle suspension was sonicated for $1 \mathrm{~min}$ and then extruded successively through 1.0, 0.4, 0.2 and $0.1 \mu \mathrm{m}$ polycarbonate membranes (Whatman, Nucleopore Track-Etch Membrane) at $50{ }^{\circ} \mathrm{C}$ to obtain SUVs. The GalNAc content was determined by heating a mixture of SUVs $(50 \mu \mathrm{l})$ and aqueous TFA $(2 \mathrm{M}, 200 \mu \mathrm{l})$ in a sealed tube for $4 \mathrm{~h}$ at $100{ }^{\circ} \mathrm{C}$. The solution was then concentrated in vacuo and analyzed by high-pH anion exchange chromatography using a pulsed amperometric detector (Methrome) and CarboPac columns PA-10 and PA-20 (Dionex).

Dose and immunization schedule. Groups of five mice (female BALB/c, age 8-10 weeks, from Jackson Laboratories) were immunized four or five times at 1-week intervals. Each boost included $3 \mu \mathrm{g}$ of saccharide in the liposome formulation. In some immunizations, the external immunoadjuvant QS-21 (10 $\mu \mathrm{g}$; Antigenics Inc.) was included. Serum samples were obtained before immunization (prebleed) and 1 week after the final immunization. The final bleeding was done by cardiac bleed.

Serologic assays. Anti-MUC1 IgG, IgG1, IgG2a, IgG2b and IgG3 antibody titers were determined by ELISA, as described previously ${ }^{5}$. Briefly, ELISA plates (Thermo Electron Corp.) were coated with a conjugate of the MUC1 glycopeptide conjugated to BSA through a bromoacetyl linker (BSA-BrAcMUC1). Serial dilutions of the sera were allowed to bind to immobilized MUC1. Detection was accomplished by the addition of phosphate-conjugated anti-mouse IgG (Jackson ImmunoResearch Laboratories Inc.), IgG1 (Zymed), IgG2a (Zymed), IgG2b (Zymed) or IgG3 (BD Biosciences Pharmingen) antibodies. After addition of $p$-nitrophenyl phosphate (Sigma), the absorbance was measured at $405 \mathrm{~nm}$ with wavelength correction set at $490 \mathrm{~nm}$ using a microplate reader (BMG Labtech). The antibody titer was defined as the highest dilution yielding an optical density of 0.1 or greater over that of normal control mouse sera.

Flow cytometry analysis. Pre- and postimmunization sera were diluted 50-fold and incubated with MCF7 and SK-MEL-28 single-cell suspensions for $30 \mathrm{~min}$ on ice. Next, the cells were washed and incubated with goat anti-mouse IgG $\gamma$-chain-specific antibody conjugated to fluorescein isothiocyanate (FITC; Sigma) for $20 \mathrm{~min}$. Cells were analyzed by flow cytometry using the FACSCalibur flow cytometer (Becton Dickinson Immunocytometry Systems), and data analysis was performed with FlowJo software (Tree Star, Inc.).

Binding and uptake assay. RAW $264.7 \gamma \mathrm{NO}(-)$ cells, HEK 293T cells and HEK 293T cells stably transfected with mouse TLR2, TLR2 plus TLR6 or TLR4 plus CD14 and MD2 $\left(2.6 \times 10^{6}\right.$ cells ml $\left.^{-1}\right)$ were exposed to Alexa Fluor 488 -labeled compound $11\left(1 \mu \mathrm{g} \mathrm{ml}^{-1}\right)$ for $30 \mathrm{~min}$ at $37^{\circ} \mathrm{C}$. Cells were harvested and washed in HNE buffer (HEPES, $20 \mathrm{mM}$; NaCl, $150 \mathrm{mM}$; EDTA, $1 \mathrm{mM}$ ). Samples that were assessed for internalization only were treated with trypsin $\left(500 \mu \mathrm{g} \mathrm{m}^{-1}\right)$ for $1 \mathrm{~min}$ and washed in HNE buffer. Next, cells were lysed in Passive Lysis Buffer (Promega), and the fluorescence (absorbance $485 \mathrm{~nm}$, emission $538 \mathrm{~nm}$ ) of the cell lysates was measured using the POLARstar OPTIMA combination luminometer/fluorometer (BMG Labtech). Fluorescence values were normalized for maximum possible fluorescence (100\%), using untreated cell lysates spiked with the fluorescent compound. The data are presented as mean \pm s.d. of triplicate treatments, with each experiment being repeated three times.

Other methods. See Supplementary Methods for sources of reagents, cell maintenance, TNF- $\alpha$ assay, evaluation of materials for contamination by lipopolysaccharide, dendritic cell maturation, transfection and NF- $\mathrm{\kappa B}$ activation assay.

Note: Supplementary information and chemical compound information is available on the Nature Chemical Biology website.

\section{ACKNOWLEDGMENTS}

We thank L. Jaso-Friedmann for helpful discussions and G.K. Lewis for the dendritic cell maturation measurements. This research was supported by the National Cancer Institute of the US National Institutes of Health (Grant No. RO1 CA88986).

\section{COMPETING INTERESTS STATEMENT}

The authors declare no competing financial interests.

Published online at http://www.nature.com/naturechemicalbiology Reprints and permissions information is available online at http://npg.nature.com/ reprintsandpermissions

1. Springer, G.F. Immunoreactive $T$ and $T n$ epitopes in cancer diagnosis, prognosis, and immunotherapy. J. Mol. Med. 75, 594-602 (1997).

2. Dube, D.H. \& Bertozzi, C.R. Glycans in cancer and inflammation. Potential for therapeutics and diagnostics. Nat. Rev. Drug Discov. 4, 477-488 (2005).

3. Ouerfelli, O., Warren, J.D., Wilson, R.M. \& Danishefsky, S.J. Synthetic carbohydratebased antitumor vaccines: challenges and opportunities. Expert Rev. Vaccines 4 , 677-685 (2005).

4. Slovin, S.F., Keding, S.J. \& Ragupathi, G. Carbohydrate vaccines as immunotherapy for cancer. Immunol. Cell Biol. 83, 418-428 (2005).

5. Buskas, T., Li, Y.H. \& Boons, G.J. The immunogenicity of the tumor-associated antigen Lewis(y) may be suppressed by a bifunctional cross-linker required for coupling to a carrier protein. Chem. Eur. J. 10, 3517-3524 (2004).

6. Ni, J., Song, H., Wang, Y., Stamatos, N.M. \& Wang, L.X. Toward a carbohydrate-based HIV-1 vaccine: synthesis and immunological studies of oligomannose-containing glycoconjugates. Bioconjug. Chem. 17, 493-500 (2006).

7. Reichel, F., Ashton, P.R. \& Boons, G.J. Synthetic carbohydrate-based vaccines: synthesis of an L-glycero-D-manno-heptose antigen-T-epitope-lipopetide conjugate. Chem. Commun. 21, 2087-2088 (1997).

8. Alexander, J. et al. Linear PADRE T helper epitope and carbohydrate B cell epitope conjugates induce specific high titer IgG antibody responses. J. Immunol. 164, 16251633 (2000).

9. Kudryashov, V. et al. Toward optimized carbohydrate-based anticancer vaccines: epitope clustering, carrier structure, and adjuvant all influence antibody responses o Lewis(y) conjugates in mice. Proc. Natl. Acad. Sci. USA 98, 3264-3269 (2001).

10. Lo-Man, R. et al. Anti-tumor immunity provided by a synthetic multiple antigenic glycopeptide displaying a tri-Tn glycotope. J. Immunol. 166, 2849-2854 (2001).

11. Jiang, Z.H. \& Koganty, R.R. Synthetic vaccines: the role of adjuvants in immune targeting. Curr. Med. Chem. 10, 1423-1439 (2003).

12. Jackson, D.C. et al. A totally synthetic vaccine of generic structure that targets Toll-like receptor 2 on dendritic cells and promotes antibody or cytotoxic T cell responses. Proc. Natl. Acad. Sci. USA 101, 15440-15445 (2004).

13. Lo-Man, R. et al. A fully synthetic therapeutic vaccine candidate targeting carcinomaassociated Tn carbohydrate antigen induces tumor-specific antibodies in nonhuman primates. Cancer Res. 64, 4987-4994 (2004).

14. Buskas, T., Ingale, S. \& Boons, G.J. Towards a fully synthetic carbohydratebased anticancer vaccine: synthesis and immunological evaluation of a lipidated glycopeptide containing the tumor-associated Tn antigen. Angew. Chem. Int. Ed. 44 5985-5988 (2005)

15. Dziadek, S., Kowalczyk, D. \& Kunz, H. Synthetic vaccines consisting of tumorassociated MUC1 glycopeptide antigens and bovine serum albumin. Angew. Chem. Int. Ed. 44, 7624-7630 (2005).

16. Krikorian, D., Panou-Pomonis, E., Voitharou, C., Sakarellos, C. \& Sakarellos-Daitsiotis, M. A peptide carrier with a built-in vaccine adjuvant: construction of immunogenic conjugates. Bioconjug. Chem. 16, 812-819 (2005).

17. Pan, Y., Chefalo, P., Nagy, N., Harding, C. \& Guo, Z. Synthesis and immunological properties of $\mathrm{N}$-modified GM3 antigens as therapeutic cancer vaccines. J. Med. Chem. 48, 875-883 (2005).

18. Baldus, S.E., Engelmann, K. \& Hanisch, F.G. MUC1 and the MUCs: a family of human mucins with impact in cancer biology. Crit. Rev. Clin. Lab. Sci. 41, 189-231 (2004). 
19. Leclerc, C., Deriaud, E., Mimic, V. \& van der Werf, S. Identification of a T-cell epitope adjacent to neutralization antigenic site 1 of poliovirus type 1. J. Virol. 65, 711-718 (1991).

20. Spohn, R. et al. Synthetic lipopeptide adjuvants and Toll-like receptor 2 - structureactivity relationships. Vaccine 22, 2494-2499 (2004).

21. Cato, D., Buskas, T. \& Boons, G.J. Highly efficient stereospecific preparation of Tn and TF building blocks using thioglycosyl donors and the $\mathrm{Ph}_{2} \mathrm{SO} \mathrm{Tf}_{2} \mathrm{O}$ promotor system. J. Carbohydr. Chem. 24, 503-516 (2005).

22. Metzger, J.W., Wiesmuller, K.H. \& Jung, G. Synthesis of $\mathrm{N}^{\alpha}$-Fmoc protected derivatives of S-(2,3-dihydroxypropyl)-cysteine and their application in peptide synthesis. Int. J. Pept. Protein Res. 38, 545-554 (1991).

23. Ingale, S., Buskas, T. \& Boons, G.J. Synthesis of glyco(lipo) peptides by liposomemediated native chemical ligation. Org. Lett. 8, 5785-5788 (2006).

24. Kensil, C.R. Saponins as vaccine adjuvants. Crit. Rev. Ther. Drug Carrier Syst. 13, 1-55 (1996).

25. Dabbagh, K. \& Lewis, D.B. Toll-like receptors and T-helper-1/T-helper-2 responses. Curr. Opin. Infect. Dis. 16, 199-204 (2003).
26. Saeland, E. et al. The C-type lectin MGL expressed by dendritic cells detects glycan changes on MUC1 in colon carcinoma. Cancer Immunol. Immunother. 56 1225-1236 (2007).

27. Musselli, C., Livingston, P.O. \& Ragupathi, G. Keyhole limpet hemocyanin conjugate vaccines against cancer: the Memorial Sloan Kettering experience. J. Cancer Res. Clin. Oncol. 127 Suppl 2: R20-R26 (2001).

28. Kuduk, S.D. et al. Synthetic and immunological studies on clustered modes of mucin-related $\mathrm{Tn}$ and TF O-linked antigens: the preparation of a glycopeptidebased vaccine for clinical trials against prostate cancer. J. Am. Chem. Soc. 120, 12474-12485 (1998).

29. Slovin, S.F. et al. Fully synthetic carbohydrate-based vaccines in biochemically relapsed prostate cancer: clinical trial results with alpha- $\mathrm{N}$-acetylgalactosamine-O-serine/threonine conjugate vaccine. J. Clin. Oncol. 21, 4292-4298 (2003).

30. Sorensen, A.L. et al. Chemoenzymatically synthesized multimeric Tn/STn MUC1 glycopeptides elicit cancer-specific anti-MUC1 antibody responses and override tolerance. Glycobiology 16, 96-107 (2006). 